\title{
MR Imaging for Differentiating Contrast Staining from Hemorrhagic Transformation after Endovascular Thrombectomy in Acute Ischemic Stroke: Phantom and Patient Study
}

\author{
(D).-H. You, (D)B. Kim, (DB.K. Kim, and (D).-i. Suh
}

\begin{abstract}
BACKGROUND AND PURPOSE: Early differentiation of contrast staining from hemorrhagic transformation in patients with acute ischemic stroke who have undergone endovascular treatment is critical in preventing the delayed administration of antiplatelet agents. We aimed to demonstrate the usefulness of an immediate postinterventional DWI protocol including $B_{0}$ and gradient recalled-echo sequences to discriminate those 2 conditions through phantom and preliminary retrospective patient studies.
\end{abstract}

MATERIALS AND METHODS: On 3T MR imaging, the signal intensities of the phantom models consisting of iodinated contrast agents diluted with normal saline and arterial blood were compared using TTWI, T2WI, and gradient recalled-echo sequences. A total 17 patients ( 8 with hemorrhagic transformation and 9 with contrast staining; 8 men and 9 women; mean age, $72.00 \pm 10.91$ years; range, 52-90 years) who underwent mechanical thrombectomy for acute ischemic stroke and showed newly appearing hyperdense lesions on immediate ( $<24$ hours) postinterventional nonenhanced CT scans were included in this study. Immediate postinterventional DWI of patients were compared.

RESULTS: In the phantom study, iodinated contrast agents diluted with normal saline showed minimal signal drop, while those diluted with arterial blood demonstrated dark signal intensity in the T2WI and gradient recalled-echo sequences. In the patient study, all hemorrhagic transformations and none of the contrast staining demonstrated dark or low signal (<gray matter) intensities similar to those of the vessel in the $B_{0}-D W I$ and gradient recalled-echo images.

CONCLUSIONS: According to our preliminary results, contrast staining might be differentiated from hemorrhagic transformation using an immediate postinterventional DWI protocol including gradient recalled-echo sequences. It might be possible to expedite establishment of postinterventional medical treatment strategy.

ABBREVIATIONS: $\mathrm{CT}_{\mathrm{FU}}=$ postinterventional (24-72 hours) follow-up nonenhanced brain $\mathrm{CT} ; \mathrm{CT}_{\text {imme }}=$ postinterventional $(<24$ hours) nonenhanced brain $\mathrm{CT}$; GRE $=$ gradient recalled-echo; IODBL = iodinated contrast agents diluted with arterial blood; IODNS = iodinated contrast agents diluted with normal saline

A fter endovascular treatment in patients with acute ischemic stroke, newly developed parenchymal hyperdensity is frequently encountered in immediate postinterventional CT images $(31 \%-61 \%) .{ }^{1-3}$ Although a large proportion $(73 \%-79 \%)$ of the parenchymal hyperdensity represents contrast staining, the rest is associated with hemorrhagic transformation. During intra-arterial thrombolysis or thrombectomy, not only the underlying isch-

Received July 10, 2018; accepted after revision August 29.

From the Department of Radiology (S.-H.Y., B.K., B.K.K.), Korea University Anam Hospital, Seoul, Korea; and Department of Radiology (S.-i.S.), Korea University Guro Hospital, Seoul, Korea.

Please address correspondence to Byungjun Kim, MD, PhD, Department of Radiology, Anam Hospital, Korea University College of Medicine, 126-1, 5-Ka Anam-dong, Sungbuk ku, Seoul 136-705, Korea; e-mail: cardillo@hanmail.net

$\equiv$ Indicates article with supplemental on-line table.

$\checkmark$ Indicates article with supplemental on-line photos.

http://dx.doi.org/10.3174/ajnr.A5848 emic change but also the intra-arterial thrombolytics and mechanical injury could cause disruption of the blood-brain barrier. Additionally, a large number of concentrated iodinated contrast agents promote breakdown of the blood-brain barrier via chemical irritation. ${ }^{4,5}$ It is possible for contrast agents to escape from the vessels when the blood-brain barrier breaks down, but cellular components of the blood, such as red blood cells, cannot leak from the vessels. This phenomenon is called contrast staining, contrast enhancement, or contrast extravasation. ${ }^{3,5-7}$ It is not a risk factor for hemorrhagic transformation; therefore, the rapid introduction of antiplatelet agents is possible to prevent re-occlusion in patients with acute infarction. ${ }^{2,3}$ In contrast, if the basal lamina and the blood-brain barrier are degraded by more severe injury, hemorrhagic transformation may occur. Under such circumstances, antiplatelet agents should not be administered to prevent the progression of the hemorrhage. Therefore, early differentiation of contrast staining from hemorrhagic transforma- 
tion is critical to establish an optimal postinterventional treatment strategy using antiplatelet agents.

In principle, it is difficult to differentiate parenchymal hyperdensity resulting from contrast staining from hemorrhagic transformation on immediate postinterventional CT ( $<24$ hours) due to the similar Hounsfield densities of the lesions. ${ }^{1,2,8}$ Although early diagnosis of hemorrhagic transformation after endovascular treatment is crucial, it may be differentiated from contrast staining only via follow-up CT ( $24-48$ hours). In this study, we were interested in differentiating contrast staining from hemorrhagic transformation using DWI and gradient recalled-echo (GRE) imaging. The negative susceptibility and T2-shortening effect of iodinated contrast agents have been demonstrated in previous phantom and animal studies. ${ }^{9}$ However, no studies have compared the signal intensities of iodinated contrast agents diluted with normal saline (IODNS) representing contrast staining and those diluted with arterial blood (IODBL) representing hemorrhagic transformation. Additionally, the usefulness of the immediate postinterventional DWI protocol for differentiating contrast staining and hemorrhagic transformation has not yet been elucidated using patient data. The aim of the present study was to compare the MR signal intensities of iodinated contrast agents mixed with normal saline and arterial blood in a phantom model. We also aimed to validate the usefulness of an immediate postinterventional DWI protocol, including $\mathrm{B}_{0}$ and GRE imaging to discriminate contrast staining and hemorrhagic transformation using retrospective patient imaging data.

\section{MATERIALS AND METHODS Phantom Model}

Forty-two 5-mL cylindric plastic tubes were prepared for the phantom. The diameter and height of the tubes were 10 and $50 \mathrm{~mm}$, respectively. The inner walls of the tubes were coated with heparin.

Thirty-five cells of the phantom were filled with 5 different kinds of nonionic iodinated contrast agents (Visipaque, iodixanol, $320 \mathrm{I} \mathrm{mg/mL,} 2.5 \mathrm{I} \mathrm{mol} / \mathrm{L}$, iso-osmolar, GE Healthcare, Piscataway, New Jersey; Omnipaque, iohexol, $350 \mathrm{I} \mathrm{mg} / \mathrm{mL}, 2.8 \mathrm{I}$ mol/L, low-osmolar, GE Healthcare; Xenetix, iobitridol, 300 I $\mathrm{mg} / \mathrm{mL}, 2.4 \mathrm{I} \mathrm{mol} / \mathrm{L}$, low-osmolar, Guerbet, Roissy, France; Iomeron, iomeprol, $300 \mathrm{I} \mathrm{mg} / \mathrm{mL}, 2.4 \mathrm{~mol} / \mathrm{L}$, low-osmolar, Bracco, Milan, Italy; and Pamiray, iopamidol, $300 \mathrm{I} \mathrm{mg/mL,} 2.4$ mol/L, low-osmolar, Dongkook Pharm, Seoul, Korea) diluted with normal saline ( $0.9 \%$ sodium chloride) at 7 different concentrations $(0,0.1,0.4,0.6,1.2,2$, and $2.4 \mathrm{I} \mathrm{mol} / \mathrm{L})$. The remaining 7 cells were filled with one of the nonionic iodinated contrast agents (Visipaque) diluted with arterial blood at 7 different concentrations $(0$, $0.1,0.4,0.6,1.2,2$, and $2.4 \mathrm{I} \mathrm{mol} / \mathrm{L}$ ). In a previous pilot study, the aforementioned 5 nonionic iodinated contrast agents showed the same tendency toward magnetic fields regardless of their different side chains. Therefore, we diluted a representative contrast agent with human arterial blood for the phantom of IODBL.

\section{MR Imaging Acquisition and Analysis of Phantom}

The phantom with IODNS and IODBL was scanned by a 3T MR imaging unit (Tim Trio; Siemens, Erlangen, Germany) using spin-echo acquisitions with T1WI (TR/TE, 500/10 ms), T2WI (TR/TE, 6000/115 ms), and GRE (TR/TE, 535/15 ms) sequences.

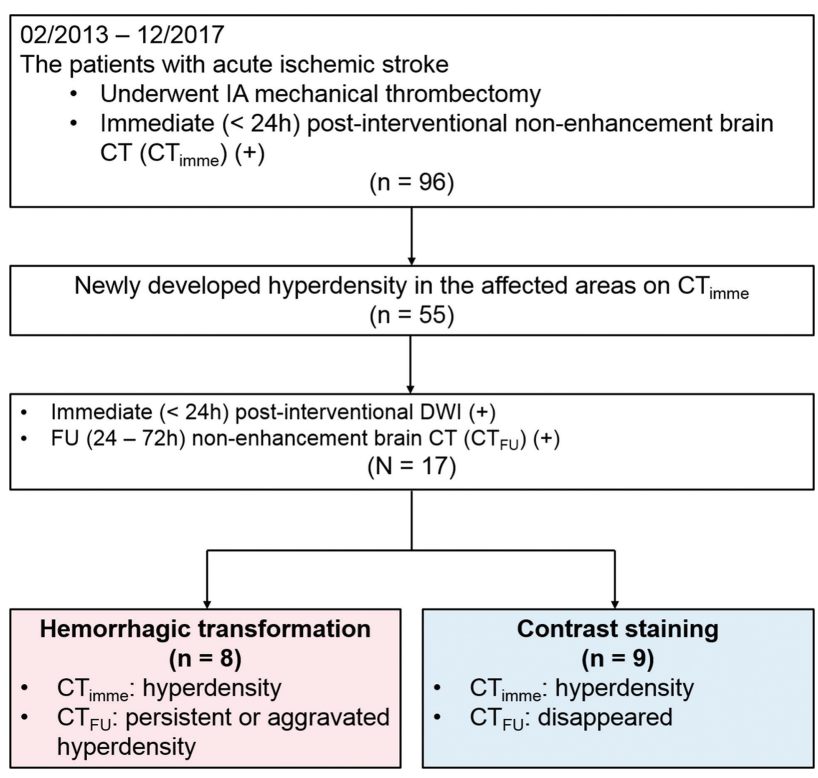

FIG 1. Study design.

The T1WI, T2WI, and GRE sequences were scanned in a visual analysis; then, another T2WI was performed after changing the TE (8.4-185 ms, TR fixed at $2500 \mathrm{~ms}, 1$ single acquired FOV of $123 \times 250 \mathrm{~mm}$, and a matrix of $256 \times 126)$ to measure the T2relaxation time. All the blood-containing tubes were replaced with new tubes containing newly sampled arterial blood and the same concentration of iodinated contrast agents to maintain the same chemical status of hemoglobin. Finally, another T1WI was acquired after changing the TR (180-250 ms, TE fixed at $8.7 \mathrm{~ms}, 1$ single acquired FOV of $123 \times 250 \mathrm{~mm}$, and a matrix of $256 \times 126$ ).

After reading the DICOM data from the MR imaging system, the T1- and T2-relaxation times for the phantom were calculated using a software for analyzing functional neuroimaging data.

\section{Patients}

This retrospective study was approved by the institutional review board of Korea University Anam Hospital. Informed consent was waived because of its retrospective nature. Between February 2013 and December 2017, ninety-six consecutive patients with ischemic stroke underwent intra-arterial mechanical thrombectomy, followed by immediate ( $<24$ hours) postinterventional nonenhanced brain $\mathrm{CT}\left(\mathrm{CT}_{\mathrm{imme}}\right)$. The Solitaire (Covidien, Irvine, California) was used as a stent-retriever system for all the patients. Among them, patients who satisfied the following criteria were included in this study: 1) patients who showed newly developed hyperdensity in the affected areas on $\mathrm{CT}_{\text {imme }}$; 2) patients who underwent DWI including $B_{0}$ and GRE sequences within 24 hours after intra-arterial mechanical thrombectomy; and 3) patients who underwent follow-up nonenhanced brain $\mathrm{CT}\left(\mathrm{CT}_{\mathrm{FU}}\right)$ between 24 and 72 hours after thrombectomy. Based on previous studies, ${ }^{5,10}$ hemorrhagic transformation was defined as a parenchymal hyperdensity in the infarcted area on $\mathrm{CT}_{\text {imme }}$ that persisted on $\mathrm{CT}_{\mathrm{FU}}$. On the other hand, contrast staining was defined as a parenchymal hyperdensity on $\mathrm{CT}_{\text {imme }}$ that had disappeared on $\mathrm{CT}_{\mathrm{FU}}$. The patients were divided into 2 groups based on the findings on $\mathrm{CT}_{\text {imme }}$ and $\mathrm{CT}_{\mathrm{FU}}$ (Fig 1). Among them, 14 patients 

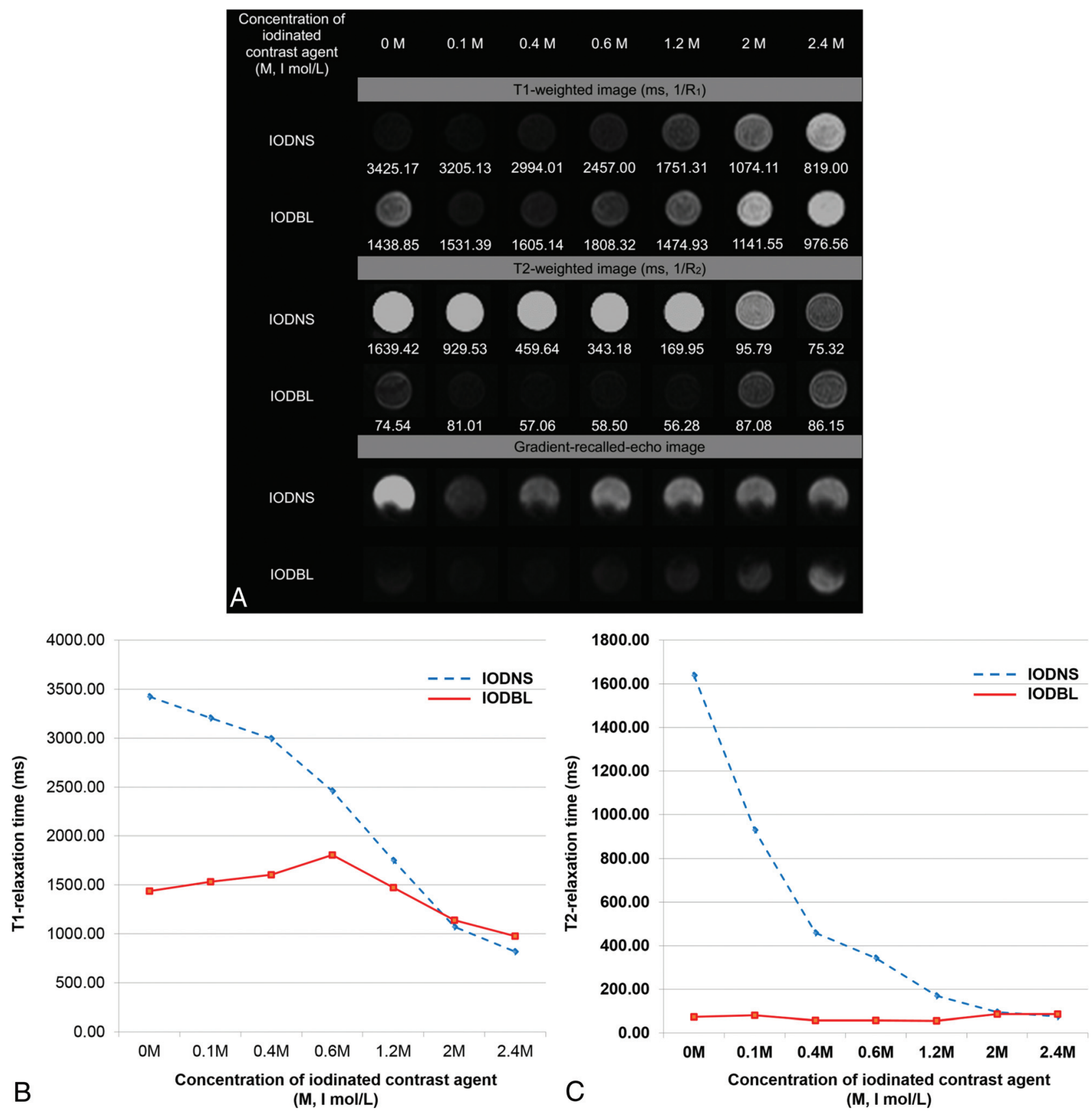

FIG 2. MR signal intensity of IODNS and IODBL on T1-weighted, T2-weighted, and gradient recalled-echo images. A, Images from the phantom. $B$, TT-relaxation times of IODNS and IODBL at different concentrations. C, T2-relaxation times of IODNS and IODBL according to the concentration of iodinated contrast agents. Visipaque (iodixanol, $320 \mathrm{I} \mathrm{mg} / \mathrm{mL}, 2.5 \mathrm{I} \mathrm{mol} / \mathrm{L}$, iso-osmolar) was used as the iodinated contrast agent in this phantom.

(14/17 [82.35\%]) also underwent conventional MR imaging, including T1WI and T2WI within 24 hours after intra-arterial mechanical thrombectomy. The type of the hemorrhagic transformation was evaluated on the basis of the European-Australasian Acute Stroke Study (ECASS) criteria. ${ }^{11}$ : Symptomatic intracerebral hemorrhage was defined according to ECASS II. ${ }^{12}$ The fate of contrast staining was analyzed using the last imaging study before the discharge.

\section{MR Imaging Acquisition and Analysis of Patients}

MR images were acquired using four 3T MR imaging scanners (Prisma, Skyra, and Trio Tim, Siemens; and Achieva, Philips Healthcare, Best, the Netherlands) with a 32- or 64-channel head coil. Imaging sequences included DWI $\left(\mathrm{B}_{0}, b=1000\right)$, GRE imaging, T2WI, and T1WI. The MR imaging parameters were as follows: single-shot echo-planar imaging sequence; TR/TE/matrix $=3590-5970 \mathrm{~ms} / 57-91 \mathrm{~ms} / 128 \times 120-192 \times 192, b=0$ and $1000 \mathrm{~s} / \mathrm{mm}^{2}$, bandwidth $=920-2374 \mathrm{~Hz}$ per voxel, 3 signals acquired for DWI; TR/TE/flip angle/matrix $=543-819 \mathrm{~ms} / 12-15$ $\mathrm{ms} / 18^{\circ}-20^{\circ} / 244 \times 244-320 \times 240$ for GRE imaging; TR/TE/flip angle $/$ matrix $=3000-6450 \mathrm{~ms} / 80-118 \mathrm{~ms} / 90^{\circ}-150^{\circ} / 368 \times 353-$ $512 \times 358$ for spin-echo T2WI; TR/TE/flip angle/matrix $=578-$ $1850 \mathrm{~ms} / 10-12 \mathrm{~ms} / 90^{\circ}-120^{\circ} / 224 \times 224-384 \times 269$ for fast spinecho T1WI; section thickness, $4-5 \mathrm{~mm}$ (with a gap of $0.5-0.8$ $\mathrm{mm})$; and $\mathrm{FOV}=179 \times 220$ or $220 \times 220 \mathrm{~mm}$. 
Table 1: MR signal intensities of hemorrhagic transformation and contrast staining in $B_{0}$ and GRE image

\begin{tabular}{ccccc}
\hline & $\begin{array}{c}\text { Hemorrhagic } \\
\text { Transformation }(\boldsymbol{n}=8)\end{array}$ & $\begin{array}{c}\text { Contrast } \\
\text { Staining }(\boldsymbol{n}=9)\end{array}$ & $\begin{array}{c}\text { Total } \\
(\boldsymbol{n}=17)\end{array}$ & $\boldsymbol{P}$ \\
\hline $\mathrm{B}_{0}$ & & & & $<.001$ \\
Score 1 & $8(100.00 \%)$ & $0(0.00 \%)$ & $8(47.06 \%)$ & \\
Score 2 & $0(0.00 \%)$ & $0(0.00 \%)$ & $0(0.00 \%)$ & \\
Score 3 & $0(0.00 \%)$ & $3(33.33 \%)$ & $3(17.65 \%)$ & \\
Score 4 & $0(0.00 \%)$ & $6(66.67 \%)$ & $6(35.29 \%)$ & $<.001$ \\
GRE & & & & \\
Score 1 & $8(100.00 \%)$ & $0(0.00 \%)$ & $8(47.06 \%)$ & \\
Score 2 & $0(0.00 \%)$ & $0(0.00 \%)$ & $0(0.00 \%)$ & \\
Score 3 & $0(0.00 \%)$ & $6(66.67 \%)$ & $6(35.29 \%)$ & \\
Score 4 & $0(0.00 \%)$ & $3(33.33 \%)$ & $3(17.65 \%)$ & \\
\hline
\end{tabular}

${ }^{a}$ Values are numbers of patients with percentages in parentheses. Score 1, signal intensity was similar to that of the vessel; score 2, signal intensity was lower than that of the gray matter; score 3, signal intensity was similar to that of the gray matter; score 4 , signal intensity was higher than that of the gray matter.

Table 2: MR signal intensities of hemorrhagic transformation and contrast staining in TIWI and $\mathrm{T} 2 \mathrm{WI} \mathrm{I}^{\mathrm{a}}$

\begin{tabular}{ccccc}
\hline & $\begin{array}{c}\text { Hemorrhagic } \\
\text { Transformation }(\boldsymbol{n}=6)\end{array}$ & $\begin{array}{c}\text { Contrast } \\
\text { Staining }(\boldsymbol{n}=8)\end{array}$ & $\begin{array}{c}\text { Total } \\
(\boldsymbol{n}=14)\end{array}$ & $\boldsymbol{P}$ \\
\hline TIWI & $1(16.67 \%)$ & $0(0.00 \%)$ & $1(7.14 \%)$ & .042 \\
Score 1 & $5(83.33 \%)$ & $3(37.50 \%)$ & $8(57.14 \%)$ & \\
Score 2 & $0(0.00 \%)$ & $5(62.50 \%)$ & $5(35.71 \%)$ & \\
Score 3 & $0(0.00 \%)$ & $0(0.00 \%)$ & $0(0.00 \%)$ & .001 \\
Score 4 & & & & \\
T2WI & $6(100.00 \%)$ & $0(0.00 \%)$ & $6(42.86 \%)$ & \\
Score 1 & $0(0.00 \%)$ & $0(0.00 \%)$ & $0(0.00 \%)$ & \\
Score 2 & $0(0.00 \%)$ & $3(37.50 \%)$ & $3(21.43 \%)$ & \\
Score 3 & $0(0.00 \%)$ & $5(62.50 \%)$ & $5(35.71 \%)$ & \\
Score 4 & & &
\end{tabular}

${ }^{a}$ Values are numbers of patients with percentages in parentheses. Score 1, signal intensity was similar to that of the vessel; score 2, signal intensity was lower than that of the gray matter; score 3, signal intensity was similar to that of the gray matter; score 4 , signal intensity was higher than that of the gray matter.

The imaging analyses for dividing the groups were performed by 1 neuroradiologist (S.-H.Y. with 7 years of clinical experience) using the $\mathrm{CT}_{\text {imme }}$ and $\mathrm{CT}_{\mathrm{FU}}$ images. Analyses of the immediate postinterventional MR images were performed by 2 neuroradiologists (B K.K. and B.K. with 8 and 13 years of clinical experience, respectively) who were blinded to the $\mathrm{CT}_{\mathrm{FU}}$ data. $\mathrm{B}_{0}, \mathrm{DWI}$, and GRE images of all patients were analyzed and T1WI/T2WI of those who underwent conventional MR imaging were reviewed. MR images, $\mathrm{CT}_{\text {imme }}$ images, and information concerning the target location were provided to the 2 reviewers. Two reviewers performed visual analysis based on the following criteria that we devised for the study: score 1, signal intensity similar to that of the vessel; score 2, signal intensity lower than that of the gray matter; score 3, signal intensity similar to that of the gray matter; and score 4 , signal intensity higher than that of the gray matter.

\section{Statistical Analysis}

The Mann-Whitney $U$ test was used to compare the medians of continuous variables that were nonparametrically distributed (NIHSS and mRS scores) and ordinal variables (TICI scores, hemorrhagic transformation, and MR signal intensity score), while the unpaired $t$ test was used to compare the means of continuous variables (age) between the 2 groups. The Fisher exact test was used to compare categoric variables between the groups. All statistical analyses were performed using SPSS Statistics for Windows, Version 20.0 (IBM, Armonk, New York). A $P$ value $<.05$ was considered statistically significant.

\section{RESULTS}

MR Signal Intensities of Various lodinated Contrast Agents

The MR signal intensities and relaxation times of the 5 different IODNSs in the T1-weighted, T2-weighted, and GRE images are shown in On-line Figs 1-3. All 5 iodinated contrast agents showed a similar tendency of signal intensity change at each concentration. T1- and T2-shortening effects were commonly observed, which became more prominent at high iodine concentrations. At the lowest concentration $(0.1 \mathrm{I} \mathrm{mg} / \mathrm{mL})$, the $\mathrm{T} 1$ values of the 5 different IODNSs ranged from 3205 to $3704 \mathrm{~ms}$. As the concentration increased, the T1-relaxation times progressively decreased to a range of 819-1144 ms at the highest concentration (2.4 I mg/mL). The T2 values of the 5 different IODNSs ranged from 1030-1913 $\mathrm{ms}$ at the lowest concentration ( $0.1 \mathrm{I} \mathrm{mg/}$ $\mathrm{mL})$. As the concentration increased, the T2-relaxation times were progressively reduced in all the iodinated contrast agents and ranged from $52-78 \mathrm{~ms}$ at the highest concentration $(2.4 \mathrm{I} \mathrm{mg} / \mathrm{mL})$. In the GRE images, the signal intensity did not show a specific trend according to concentration of the contrast agents.

\section{Comparison of MR Signal Intensities between IODNS and IODBL}

MR signal intensities and relaxation times of IODNS and IODBL in the T1-weighted, T2-weighted, and GRE images are shown in Fig 2. At most iodine concentration ranges (0.1-1.2 I mol/L), IODNS could be differentiated from IODBL visually in the T2weighted and GRE images. On T2WI, the T2 signal intensities of IODBL were darker than those of IODNS in visual assessment. The T2-relaxation times of IODBL (56-87 ms) were lower than those of IODNS (96-1639 ms) at most iodine concentrations (range, 0.1-2.0 I mg/mL) (Fig $2 A,-B$ ). On GRE images, IODBL showed dark signal intensity and IODNS showed intermediate signal intensity. There was no specific trend of signal change according to the concentration of the contrast agent (Fig $2 A)$. On T1WI, it was impossible to differentiate IODNS and IODBL (Fig 2C).

\section{Baseline Characteristics of Patients}

The clinical characteristics of patients are summarized in On-line Table 1. Among the 17 patients, 8 were classified as having hemorrhagic transformation, and 9 , as having contrast staining. No significant difference was observed between the 2 groups. One of 8 patients $(12.50 \%)$ in the hemorrhagic transformation group showed symptomatic intracerebral hemorrhage. The final outcomes of areas of contrast staining were infarction in 7 patients (7/9 [77.78\%]) (Online Table 2). 
bo

GRE
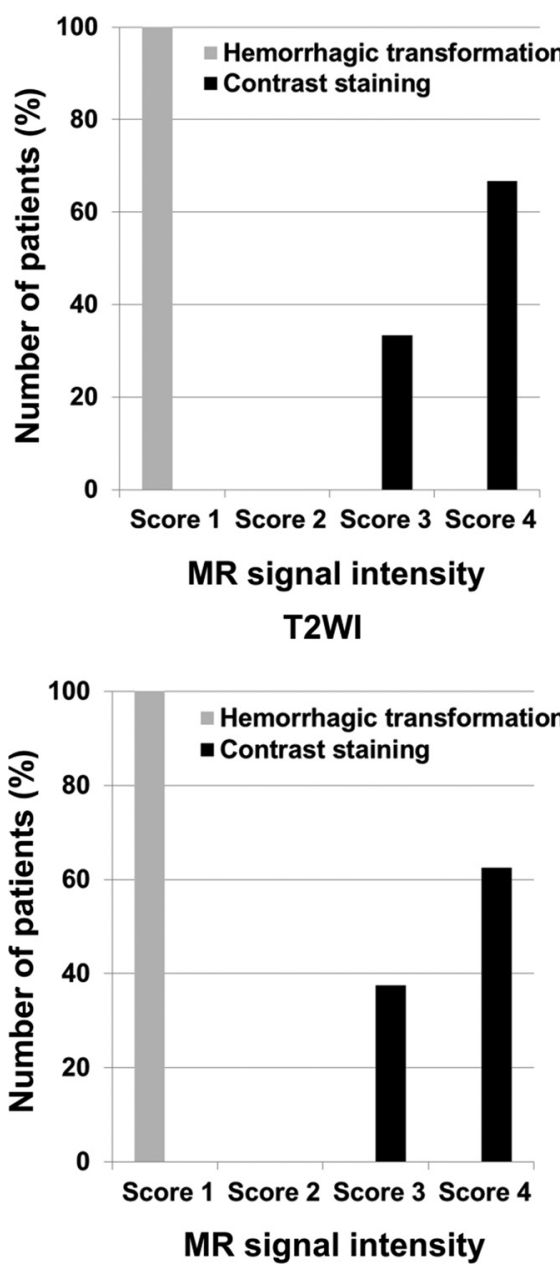

FIG 3. Magnetic signal intensity score of hemorrhagic transformation and contrast staining on $B_{0}$, gradient recalled-echo, T2-weighted, and T1-weighted imaging. Score 1, signal intensity was similar to that of the vessel; score 2, signal intensity was lower than that of the gray matter; score 3, signal intensity was similar to that of the gray matter; and score 4, signal intensity was higher than that of the gray matter.

\section{MR Signal Intensities of Hemorrhagic Transformation and Contrast Staining}

The MR signal intensity scores of hemorrhagic transformation and contrast staining are summarized in Tables 1 and 2 and Fig 3. Hemorrhagic transformation showed significantly lower visual scores than contrast staining in all MR imaging sequences $\left(B_{0}\right.$, GRE, T1WI, and T2WI $)(P<.05)$. All hemorrhagic transformations had a score of 1 (signal intensity was similar to that of the vessel) on $\mathrm{B}_{0}$, GRE, and T2WI. However, all contrast staining had scores of 3 or 4 (signal intensity was similar to or higher than that of the gray matter). Representative MR images, including $B_{0}$, GRE, T2WI, and T1WI, are shown in Fig 4. Hemorrhagic transformation showed a dark signal intensity similar to that of the vessel, and contrast staining demonstrated an iso- or hyper-signal intensity compared with that of the normal gray matter on $B_{0}$, GRE, and T2WI.

\section{DISCUSSION}

In the present phantom study, the T2WI and GRE sequences could differentiate IODBL from IODNS at most of the iodine concentrations $(0.1-1.2 \mathrm{~mol} / \mathrm{L})$. In the exploratory patient study, immediate postinterventional $\mathrm{B}_{0}$ and GRE images enabled differentiation of contrast staining from hemorrhagic transformation in patients with acute ischemic stroke who underwent endovascular thrombectomy.

The use of DWI for differentiating contrast staining from hemorrhagic transformation has 3 clinical advantages: First, MR imaging is available in almost all hospitals that treat acute ischemic stroke, and DWI and GRE are widely used sequences. Although the usefulness of dual-energy CT for differentiating these entities has been revealed in several previous studies, it cannot be applied in some types of CT scanners. ${ }^{6,7,13}$ Second, $B_{0}-$ DWI may be used as an alternative to T2WI with a shorter scan time and at a lower cost. Our results demonstrated that $\mathrm{B}_{0}$ imaging was not inferior to T2WI in differentiating hemorrhagic transformation and contrast staining. Third, $b=1000-D W I$ could offer information about final infarction size, which influences treatment outcomes of endovascular thrombectomy.

In T2WI and $\mathrm{B}_{0}-\mathrm{DWI}$, contrast staining could be differentiated from hemorrhagic transformation. IODNS demonstrated a T2-shortening effect, but IODBL showed a much stronger T2-shortening effect. These results correspond well with those of earlier studies. ${ }^{9,14,15}$ The mechanism of the T2shortening effect of the iodinated contrast agent is not well-known, but the bound water effect and dipole-dipole interaction theories have been suggested as the most important mechanisms. ${ }^{16,17}$ In general, the T2-shortening effect is explained by 3 major mechanisms: paramagnetism, bound water effect, and dipole-dipole interaction. ${ }^{18}$ The paramagnetic effect could be excluded because iodinated contrast agents are classified as diamagnetic materials that do not have unpaired electrons. ${ }^{19}$ The bound water effect could be the cause of the T1- and T2-shortening effects. This is because neither the iodine nor the benzene ring could influence the relaxation process of water molecules; however, the hydrophilic hydroxyl-containing portion of the iodinated contrast agent can form a hydrogen bond with water. ${ }^{19}$ Bound water shows more rapid relaxation than free water; therefore, the bound water effect of the contrast agent may lead to T2-shortening. ${ }^{18}$ A dipole-dipole interaction that occurs between 2 adjacent molecules that have different degrees of electronegativity could influence T2-shortening because iodinated contrast agents and water have different degrees of electronegativity. In the phantom study, IODBL showed a much 


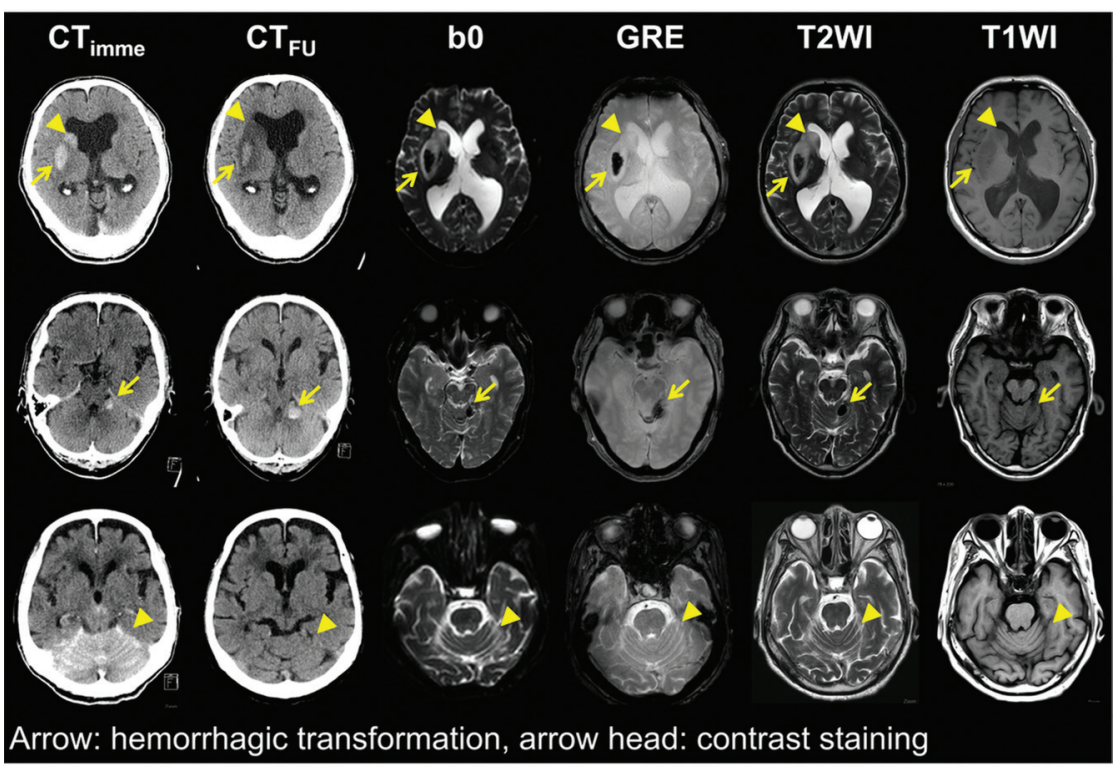

FIG 4. Representative images of hemorrhagic transformation and contrast staining in 3 patients with acute ischemic stroke. First row: images of a 66-year-old man with infarction in the territory of the right middle cerebral artery. A persistent hyperdense lesion in the right lentiform nucleus, presumed to be a hemorrhagic transformation (arrow), shows dark signal intensity in the $\mathrm{B}_{0}$, gradient recalled-echo, and T2-weighted images. In contrast, a hyperdense lesion in the right caudate nucleus, which is washed out on the follow-up nonenhanced brain CT and presumed to be contrast staining (arrowhead), demonstrates a high signal intensity in the $B_{0}$, GRE, and T2weighted images. This patient was grouped into the hemorrhagic transformation group, and the lesion in the lentiform nucleus was analyzed. Middle row: images of a 68-year-old man with infarction in the region of the right superior cerebellar artery. A persistent hyperdense lesion in the left cerebellum, presumed to be hemorrhagic transformation (arrow), shows dark signal intensity in the $\mathrm{B}_{0}$, GRE, and T2-weighted images. Lower row: images of an 88-year-old woman with bilateral cerebellar infarction. A hyperdense lesion in the bilateral upper cerebellum, which disappeared on $\mathrm{CT}_{\mathrm{FU}}$ and was presumed to be contrast staining (arrowhead), demonstrates iso-signal intensity in the $\mathrm{B}_{0}$, GRE, and T2-weighted images.

stronger T2-shortening effect than IODNS. These results could be explained by 2 hypotheses ${ }^{9,14,15}$ : First, the T2-shortening effect of blood caused by the paramagnetic mechanism was much stronger than that of the bound water and dipole-dipole interaction effects of the iodinated contrast agent. Second, the bound water and dipole-dipole interaction effects of the iodinated contrast agent were weaker in the blood-mixed environment than in the watermixed environment. Under these mechanisms, the more powerful T2-shortening effect of IODBL made it possible to distinguish IODNS from IODBL. In patient data, this effect also applied not only to T2WI but also to $\mathrm{B}_{0}-\mathrm{DWI}$; thus, hemorrhagic transformation and contrast staining could be differentiated using immediate postinterventional DWI.

In the GRE images, hemorrhagic transformation could be differentiated from contrast staining by its strong positive susceptibility. IODNS showed a more minimal signal drop than pure water in the phantom study. This effect did not show a specific trend according to the concentration or kind of contrast agent. This phenomenon is difficult to explain because iodinated contrast agents are classified as diamagnetic materials that do not have unpaired electrons. One hypothesis is that diamagnetic materials are repelled by magnetic fields. Therefore, these materials possibly reduce the intensity of the magnetic fields only minimally and cause a minimal signal drop. However, this minimal signal drop could not be visualized on patients' imaging data. This result agrees with that of a previous animal study. ${ }^{9}$
Because blood has strong positive susceptibility, hemorrhagic transformation was well-distinguished from contrast staining in the GRE images.

Five kinds of commonly used nonionic iodinated contrast agents were evaluated to reveal the effect of the side chains that are different in each contrast agent. Our study showed no significant difference in the $\mathrm{T} 1$ and $\mathrm{T} 2$ values among the different contrast agents at the same concentration. This is in concordance with the results of previous studies. ${ }^{9,16}$ The absence of a definite difference in the T1 and T2 values is probably because all kinds of iodinated contrast agents used in our study were nonionic. A minor difference in signal intensity among the contrast agents may be caused by their different side chains.

Several studies have reported the clinical relevance of newly developed parenchymal hyperdensity after mechanical thrombectomy, which is not associated with poor prognosis or symptomatic hemorrhage. ${ }^{3,10}$ In our study, the median mRS score at 90 days in the overall population that underwent mechanical thrombectomy $(n=86 / 96 ; 10$ patients lost to follow-up) was 3.0 (interquartile range, 2.0-5.0). It is similar to that in our final study population, which demonstrated newly developed parenchymal hyperdensity (3.0 [interquartile range 2.03.0]). In addition, only 1 of 17 cases showed symptomatic intracerebral hemorrhage in the present study. Regarding the longitudinal outcomes of areas of contrast staining, approximately $80 \%$ finally progressed to infarction in this study. This result is in close agreement with those of the other studies. ${ }^{10,20}$

Our study has several limitations. First, the number of patients included in our retrospective study was small. Large-scale prospective studies are needed to validate the findings of this study. Second, oxygen saturation was not measured in our study; hence, the exact chemical status of blood could not be presented. Third, we cannot predict the effect of anticoagulating agents, such as heparin, present in the tubes containing the blood samples on MR imaging.

\section{CONCLUSIONS}

According to our preliminary results, contrast staining might be differentiated from hemorrhagic transformation using an immediate postinterventional DWI protocol including GRE imaging.

\section{ACKNOWLEDGMENTS}

The authors would like to express their deepest appreciation to Kyung-Sook Yang, professor in the department of biostatistics, who provided verification regarding our statistical methods. 


\section{REFERENCES}

1. Payabvash S, Qureshi MH, Khan SM, et al. Differentiating intraparenchymal hemorrhage from contrast extravasation on post-procedural noncontrast CT scan in acute ischemic stroke patients undergoing endovascular treatment. Neuroradiology 2014;56:737-44 CrossRef Medline

2. Nikoubashman O, Reich A, Gindullis M, et al. Clinical significance of post-interventional cerebral hyperdensities after endovascular mechanical thrombectomy in acute ischaemic stroke. Neuroradiology 2014;56:41-50 CrossRef Medline

3. Parrilla G, Garciá-Villalba B, Espinosa de Rueda M, et al. Hemorrhage/contrast staining areas after mechanical intra-arterial thrombectomy in acute ischemic stroke: imaging findings and clinical significance. AJNR Am J Neuroradiol 2012;33:1791-96 CrossRef Medline

4. Rudd MA, Johnstone M, Rabbani L, et al. Thrombolytic therapy causes an increase in vascular permeability that is reversed by 1-deamino-8-D-vasopressin. Circulation 1991;84:2568-73 CrossRef Medline

5. Yoon W, Seo JJ, Kim JK, et al. Contrast enhancement and contrast extravasation on computed tomography after intra-arterial thrombolysis in patients with acute ischemic stroke. Stroke 2004;35: 876-81 CrossRef Medline

6. Phan CM, Yoo AJ, Hirsch JA, et al. Differentiation of hemorrhage from iodinated contrast in different intracranial compartments using dual-energy head CT. AJNR Am J Neuroradiol 2012;33:1088-94 CrossRef Medline

7. Gupta R, Phan CM, Leidecker C, et al. Evaluation of dual-energy CT for differentiating intracerebral hemorrhage from iodinated contrast material staining. Radiology 2010;257:205-11 CrossRef Medline

8. Dekeyzer S, Nikoubashman O, Lutin B, et al. Distinction between contrast staining and hemorrhage after endovascular stroke treatment: one CT is not enough. J Neurointerv Surg 2017;9: 394-98 CrossRef Medline

9. Nikoubashman O, Jablawi F, Dekeyzer S, et al. MRI appearance of intracerebral iodinated contrast agents: is it possible to distinguish extravasated contrast agent from hemorrhage? AJNR Am J Neuroradiol 2016;37:1418-21 CrossRef Medline
10. Lummel N, Schulte-Altedorneburg G, Bernau C, et al. Hyperattenuated intracerebral lesions after mechanical recanalization in acute stroke. AJNR Am J Neuroradiol 2014;35:345-51 CrossRef Medline

11. Fiorelli M, Bastianello S, von Kummer Rd, et al. Hemorrhagic transformation within 36 hours of a cerebral infarct: relationships with early clinical deterioration and 3-month outcome in the European Cooperative Acute Stroke Study I (ECASS I) cohort. Stroke 1999;30: 2280-84 CrossRef Medline

12. Larrue V, von Kummer R, Müller A, et al. Risk factors for severe hemorrhagic transformation in ischemic stroke patients treated with recombinant tissue plasminogen activator: a secondary analysis of the European-Australasian Acute Stroke Study (ECASS II). Stroke 2001;32:438-41 CrossRef Medline

13. Tijssen $M$, Hofman $P$, Stadler A, et al. The role of dual energy CT in differentiating between brain haemorrhage and contrast medium after mechanical revascularisation in acute ischaemic stroke. Eur Radiol 2014;24:834-40 CrossRef Medline

14. Morales $\mathrm{H}$, Lu A, Kurosawa $\mathrm{Y}$, et al. Variable MR and pathologic patterns of hemorrhage after iodinated contrast infusion in MCA occlusion/reperfusion model. J Neurointerv Surg 2017;9:1248-52 CrossRef Medline

15. Yedavalli V, Sammet S. Contrast extravasation versus hemorrhage after thrombectomy in patients with acute stroke. J Neuroimaging 2017;27:570-76 CrossRef Medline

16. Hergan K, Doringer W, Längle M, et al. Effects of iodinated contrast agents in MR imaging. Eur J Radiol 1995;21:11-17 CrossRef Medline

17. Jinkins JR, Robinson JW, Sisk L, et al. Proton relaxation enhancement associated with iodinated contrast agents in MR imaging of the CNS. AJNR Am J Neuroradiol 1992;13:19-27 Medline

18. Boulby PA, Rugg-Gunn F. T2: The Transverse Relaxation Time. West Sussex: John Wiley \& Sons; 2003

19. Bae KT. Intravenous contrast medium administration and scan timing at CT: considerations and approaches. Radiology 2010;256: 32-61 CrossRef Medline

20. Amans MR, Cooke DL, Vella M, et al. Contrast staining on CT after DSA in ischemic stroke patients progresses to infarction and rarely hemorrhages. Interv Neuroradiol 2014;20:106-15 CrossRef Medline 